\title{
Scientific Publications and Patenting by Companies: a Study of the Whole Population of Canadian Firms over 25 years
}

\author{
Forthcoming in Science and Public Policy
}

\begin{abstract}
Éric Archambault
Science-Metrix; Observatoire des sciences et des technologies (OST) and Centre interuniversitaire de recherche sur la science et la technologie (CIRST), Université du Québec à Montréal, Montréal (Québec), Canada. Email: eric.archambault@science-metrix.com
\end{abstract}

\section{Vincent Larivière}

Observatoire des sciences et des technologies (OST) and Centre interuniversitaire de recherche sur la science et la technologie (CIRST), Université du Québec à Montréal, Montréal (Québec), Canada. Email: lariviere.vincent@uqam.ca

\begin{abstract}
There is evidence in the literature that technological inventions comprise an increasing connection to scientific knowledge. This raises two related questions: 1) Are firms increasingly conducting scientific basic research? 2) Is being at the scientific forefront helping firms also to be at the technological frontier? This paper examines scientific output, as measured by numbers of papers, and technological output, as measured by patents granted to Canadian firms, during the 1980 to 2005 period. Though the number of firms publishing papers and obtaining patents is increasing, scientific research and patenting by Canadian firms are at near "homeopathic" levels. Firms that both publish papers and obtain patents 1) perform research that is more basic than firms that only publish scientific papers, 2) publish in more highly cited journals than firms that only perform scientific research; 3) publish papers that are more highly cited; 4) hold patents that are more frequently cited.
\end{abstract}

\section{Introduction}

According to Narin, Hamilton and Olivastro (1997), there is a growing link in the US between technology and public science. Technological inventions have strong connections with scientific knowledge as exemplified by the increasing number of citations to scientific papers in the prior art sections of granted patents. If the trend is for enterprises to increasingly cite science in their research, two questions are raised: 1) Are firms increasingly conducting scientific (and more basic) research? 2) Are firms conducting scientific research also obtaining intellectual property protection in the form of patents?

While there is an abundant literature on why firms apply for patents, the literature on why they perform scientific research and why they publish their results is thinner. There is a wide consensus that firms want to obtain patents in order to help secure a monopoly position, which is subsequently translated in economic benefits. A corollary would be that firms are reluctant to publish their results because placing their knowledge into the public domain denies them a monopoly over this knowledge, and result in a subsequent loss of economic competitiveness. The literature has proposed several reasons for this apparent anomaly. Regardless of the precise reasons for conducting basic research, firms must be expecting an economic return of some sort, most likely from increased competitiveness. These important issues are examined in this paper through a systematic investigation of the published papers and patents granted to the whole population of Canadian firms between 1980 and 2005.

The first part of the paper succinctly examines why firms would be performing basic scientific research and publishing their results. The second part describes the methods, the third presents the results of this study, and the fourth part is a discussion.

\section{Why do firms perform basic research?}

The link between science and technology ( $\& \& \mathrm{~T})$ in private firms became institutionalised in the second half of the $19^{\text {th }}$ century, most notably when German firms in the aniline dye industry started to systematically hire university-trained chemists (Beer, 1958). Despite the long-standing debate on the 
precise interactions between $S \& T^{1}$, and the worthy contributions still being made today on this subject ${ }^{2}$, it is undisputed that the modern industrial fabric would be entirely different if it were not for the employment by industry of university graduates who engage in a wide range of scientific activity, ranging from highly applied and goal-oriented research, to advanced and basic research.

Although the reasons for performing applied research, and R\&D more generally, have been explored at great length by economists and historians of S\&T, relatively less attention has been given to why firms perform basic scientific research. As Rosenberg (1990) notes, there appear to be major disincentives to performing basic research for firms. The first is the imperfect "appropriability" of science and the related fact that social returns are significantly higher than private returns (Nelson, 1959). For instance, the disclosure of research results by firms through peer-reviewed scientific publications increases social returns but at the expense of private returns.

Rosenberg proposes several reasons as to why firms fund and perform basic research. The first is to gain first-mover advantage: despite imperfect appropriability, being first to start the learning curves provides opportunities to create barriers to entry for followers. The application of results from basic research and securing of patent protection allows the downstream market position to be consolidated. The second reason is that it is generally large firms that engage in basic research, and they can be confident that the findings will be put to good commercial use across a diverse range of products and commercial networks. Another factor that should be considered is the unexpected and unplanned aspect of firms' activities. Indeed, while performing more applied research, they might make basic discoveries. For example, there was certainly a degree of serendipity in the discoveries of Louis Pasteur, who opened the door to the modern science of bacteriology, and of Karl Jansky's discovery of cosmic background radiation, which he made while working for the Bell Laboratories, trying to identify the cause of statics in transatlantic radiotelephone services.

One of the most powerful reasons for conducting scientific research is that it allows entry into information networks. This serves two functions. Firstly, as von Hippel (1987) pointed out in the case of collaboration between rivals, to obtain information from a network, it is necessary to be a contributor to the work of the network (see also Harhoff, Henkel and von Hippel, 2003). Secondly, firms need to perform basic research to be able to absorb the knowledge that resides on the not-so-freely-available 'information shelf. For instance, "a firm is much less likely to benefit from university research unless it also performs some basic research" (Rosenberg, 1990: 171; Mowery, 1983, Furukawa and Goto, 2006).

Rosenberg also suggested that firms often perform basic research in order to give them a better understanding of how and where to conduct research of a more applied nature. In addition, conducting basic research allows them to monitor and evaluate research conducted elsewhere. Finally, and it should be noted that this may apply more specifically to the US given the weight of its military-industrial complex, Rosenberg suggests that firms perform basic research in order to increase their visibility and eligibility for military procurement contracts.

\section{Why, then, would firms divulge the results of their research?}

One interesting hypothesis as to why firms publish the results of their scientific research is that if they encounter a research bottleneck, they may be keen to signal to the wider research community that a scientific problem is currently unsolved ${ }^{3}$. This would help bring attention to the problem and to collectively find a solution. Another hypothesis is that firms that are behind in the patent race publish papers in order to change the prior art in the hope of slowing down competitors (see e.g. Bar, 2006). Alternatively, firms sometimes publish the results of their scientific research when in the course of it they make serendipitous discoveries. These discoveries may appear to firms are being too big to be kept secret; the knowledge created would confer much smaller economic returns than the longer-term positive effects of adding to their reputation. The important aspect of this suggestion is that firms will trade the short-

\footnotetext{
${ }^{1}$ See Technology and Culture (1965) vol. 6 no. 4 for the papers by De Solla Price and Multhauf and commentaries by Beer and Condit.

2 See, e.g., Narin and Olivastro (1992); Meyer (2000).

3 This idea was suggested by our colleague Jean-Pierre Robitaille.
} 
term gains potentially associated with proprietary knowledge against the gains to be achieved from improving their reputation. Muller and Pénin’s (2006) work supports this explanation.

For Muller and Pénin, firms that openly disclose knowledge are motivated by concerns related to reputation and the benefits of having the reputation of being an innovator are huge. For Muller and Pénin, a good reputation may facilitate access to financing, grants and subsidies, and may help to win contracts. It may play an important role in increasing innovative capability by enabling firms to attract the best researchers and find excellent partners with whom to collaborate on R\&D projects. An excellent reputation and being at the forefront of science may, as Rosenberg, and Muller and Pénin suggested, make them attractive to the best academic and industrial partners and, we may add, to government scientists.

Thus, firms that perform basic scientific research might do so partly for internal reasons, such as increasing their stock of knowledge, which will allow them to develop their own products, but might also do it to orient their more applied research, understand what their competitors are doing and give them the capability to absorb knowledge generated elsewhere. When they openly disclose knowledge, through scientific publication, they do so, in many instances, to improve reputation and, over a longer time period, to gain economic and technological advantages. In this context, it is relevant to examine whether firms at the forefront of science are also at the technological frontier, since this would lend credence to the idea that performing scientific research increases competitiveness through the mediation of complex processes, such as deriving benefits from an increase in reputation.

\section{Methods}

A number of indicators are used in this paper to measure the scientific and technological impact of research. A widely used measure of the scientific impact of research is the number of citations received in subsequent papers (Garfield, 1979). This paper uses citation counts to examine whether firms are located at the scientific forefront. More specifically, it uses a variant of citation counts termed the average of relative citations (ARC), which reflects the fact that citation patterns are different in each field and subfield-e.g. there are more references per papers in biomedical research than in mathematics. The ARC is obtained by dividing the number of citations received by each paper by the average number of citations received by papers in its particular subfield for the same publication year (RC). ${ }^{4}$ The ARC of a given entity was computed using the average $\mathrm{RC}$ for each paper belonging to it.

The present paper also uses the Journal Impact Factor produced by Thomson Scientific as it can be seen as an indicator of the prestige of journals in the scientific community: the more a journal is cited, the more prestigious the journal is. Despite its well known limitations (see e.g. Archambault and Larivière, 2007; Vinkler, 2004), the Journal Impact Factor is a useful metrics to determine whether firms can be seen to derive some prestige from the publication of scientific research activities. This paper uses a variant of the impact factor (IF) termed the average relative impact factor (ARIF). This indicator is preferred to raw citation count for the same reason that the ARC metrics is preferred to use a simple average of impact factor (i.e. interfield variations in citation patterns). The ARIF of a given entity was computed using the average RIF for each paper belonging to it. When the ARC, or the ARIF, is above 1, it means that an entity (e.g. a country, a firm) scores better than the world average; when it is below 1, an entity publishes papers that are not cited as often as the world average or are not published in journals that are cited as often as the average of scientific papers considering the specialties of each paper (logarithmic transformations are sometimes used in this paper, in which case the baseline becomes 0 ).

In this paper, firms are considered to be in the forefront of science if their ARC is above 1. This is a fair assumption because the ARC' value is greatly dependent on the scientific research performed in universities, and the performance of basic research is the raison-d'etre of universities. If firms have an aggregate ARC above the world average, then clearly they are making important scientific contributions. Secondly, firms are expected to be increasing their prestige and thus improve their reputation if they publish in prestigious journals, that is, if the ARIF value of their publications is above 1. Again, this is a fair assumption because the Impact Factor of most journals is to a large extent determined by the quality

\footnotetext{
${ }^{4}$ Journals were assigned fields and subfields using the classification used by the National Science Foundation in the Science and Engineering Indicators.
} 
of the scientific research published by university researchers whose primary output is knowledge published in scientific journals.

Another indicator that is used in this paper is the "research level" (see Narin, Pinski \& Gee, 1976; Pinski \& Narin, 1976), which distinguishes between four levels ranging from applied (level 1) to basic research (level 4). This journal-level score was determined by Narin's team at CHI Research (now The Patent Board). Here, for each paper by a firm, a score was calculated by matching the degree of appliedness of the journal in which the article was published. This is useful to examine whether firms are merely performing very applied research or if on the contrary they appear to publish the result of basic scientific research.

Counting citations to patents in the prior art sections of subsequent patents is a potent metrics that can be used to determine their technological significance (Albert et al. 1991; Hall, Jaffe and Trajtenberg, 2000; Jaffe and Trajtenberg, 2002). For each patent owned by a firm over the period under study, its citations from subsequent patents were compiled to produce an indicator of the technological impact of research conducted in industry. Here, it is assumed that, aggregately, the more highly cited patents are, the closer they are to the technological frontier. In a manner similar to the use of ARC to relativize citations to papers published in varied fields, each patent's citation is relativized by the average number of citations received by patents of the same U.S. technological class and year of issuance (patent average of relative citations - PARC). When this indicator is above 1, it means that patents from a given entity have received more citations in other patents than patents of the same technological class and year. When PARC is below 1 , it means the opposite.

This paper uses data from the US Patent and Trademark Office (USPTO) database and from Thomson Scientific's Science Citation Index (SCI). Several months of work were required to create and extensively harmonize and clean a dataset comprising the whole population of Canadian firms that had obtained at least one patent or published one paper over the 1980-2005 period. Canadian firms were identified by the presence of a Canadian address in a paper or in the assignee field of a patent. Company names were standardised, which was a somewhat challenging exercise, since this project drew on data from two entirely separate databases. This was nevertheless essential to examine the connection between scientific and technological activities.

As is the case with all datasets, the ones underpinning this paper have some limitations. The main one is that, while numbers of scientific publications written by industrial researchers in Canada are a good measure of scientific output, patents may underestimate the output of Canadian technological research since they do not necessarily measure the place where invention took place; they identify the location where the intellectual property is held (IP) (see Archambault, 2002, for a distinction between these two dimensions of patents). Another limitation of the dataset used in the present research is the fact that patents owned by independent Canadian inventors are not considered as firms' patents although, in some cases, the inventor(s) are the firms' owners (see Amesse et al., 1991).

\section{The Canadian Business Enterprise R\&D Landscape As Viewed by Output Indicators}

Our data reveal (Figure 1) that there is approximately the same proportion of firms that publish scientific papers but do not hold patents (84\%) as firms that hold patents but do not publish (87\%). Of the group of firms that published one paper or obtained one patent, only $8 \%$ both published and patented. Interestingly, firms that both obtained patents and published peer-reviewed papers had an average of 16 papers and 17 patents, which suggests that this population comprises a substantial number of large firms. By comparison, firms that only published produced only 4.4 papers on average, while firms that held patents but did not publish had 2.4 patents on average. 
Figure 1 Venn diagram of scientific and technological output of Canadian firms, 1980-2005

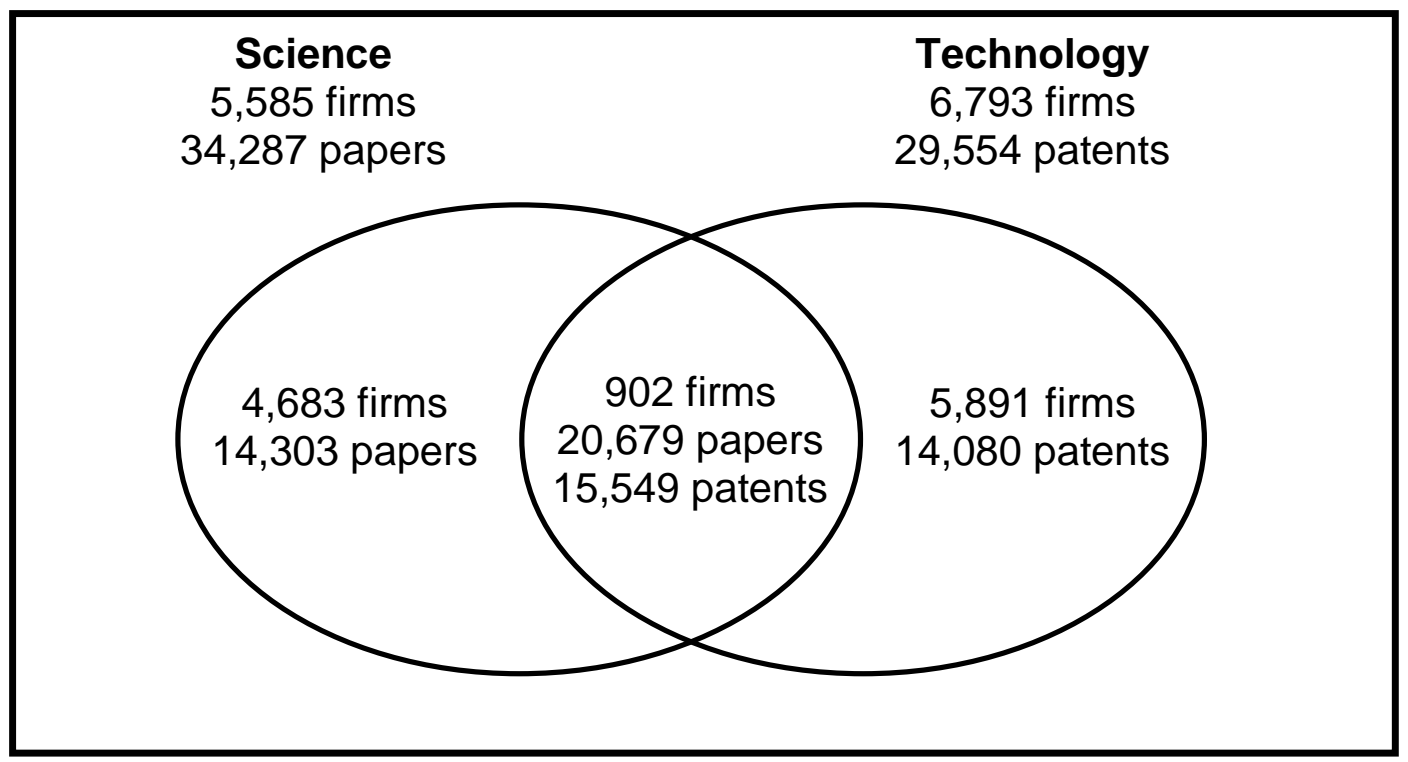

Table I shows that in contrast to the importance given to the knowledge economy discourse, Canadian firms on the whole have not entered or are far from having entered this paradigm. Overall, there were about 1 million Canadian firms in existence at any time during the last ten years. Only 1\% of Canadian firms reportedly performed R\&D in this period. Moreover, only some $0.1 \%$ of firms that were in existence in any one year published a paper or obtained a patent during that year. The percentage of firms that both published and obtained a patent during any year was $0.01 \%$ of the firms in existence. This shows that the concentration of firms that are both publishing papers and obtaining patents is nearing "homeopathic" concentrations. Hence, it is extremely rare, at least in the Canadian context, to see firms both visibly conducting basic scientific research and obtaining IP protection.

Despite these small numbers, the number of firms with a scientific or technological output grew significantly over the period studied. More precisely, while in 1983 only $0.07 \%$ of the 752,700 Canadian companies had a bibliographically measurable output (paper or patent), this share grew to $0.15 \%$ in 2003 . For firms that had both patents and papers, the concentration among firms reputed to perform R\&D grew from $0.5 \%$ to $0.9 \%$ - a significant increase but a percentage that confirms that this phenomenon is rare. It also raises the question of whether the rules used in Canada to declare that firms conduct R\&D are adequately reflecting reality or provides a smokescreen for the Canadian government to subsidize firms with tax breaks.

If one considers only Canadian firms that are reportedly active in $R \& D$ as the denominator, the relative importance of Canadian firms with one or both types of output is much higher and increased from $9 \%$ of firms in 1994 to $12 \%$ in 2002. The proportion of firms that reportedly performed R\&D but only published papers grew slightly, from $5 \%$ in 1994 to $6 \%$ in 2002. Similarly, the proportion of firms performing R\&D that obtained patents grew from 5\% in 1994 to $7 \%$ in 2002 . This shows that using only either papers or patents as indicators of activities for firms reportedly performing R\&D is not commendable since, even in the best case (when both indicators are considered), for $88 \%$ of firms no such output is measured over a 25 -year period. Thus, for most firms claiming to perform $\mathrm{R} \& \mathrm{D}$, there is necessarily an important part that is submerged below the line of sight providing by traditional bibliometric measures and it is therefore clear that other innovation indicators are required to examine the whole industrial dynamics picture, or that a stricter definition of $R \& D$ is required. 
Table I Number of Canadian firms in the science and technology landscape ${ }^{5}$

\begin{tabular}{|c|c|c|c|c|c|}
\hline Year & $\begin{array}{r}\text { Canadian } \\
\text { Frms }\end{array}$ & $\begin{array}{r}\text { Canadian Frms Active } \\
\text { in R\&D }\end{array}$ & $\begin{array}{r}\text { Frms Publishing } \\
\text { Papers }\end{array}$ & $\begin{array}{r}\text { Frms Holding } \\
\text { US Patents }\end{array}$ & $\begin{array}{r}\text { Frms with both Papers } \\
\text { and Patents }\end{array}$ \\
\hline 1994 & 918000 & 11132 & 520 & 560 & 59 \\
\hline 1995 & 923000 & 10771 & 540 & 575 & 67 \\
\hline 1996 & 925200 & 9805 & 619 & 595 & 64 \\
\hline 1997 & 945000 & 9649 & 631 & 644 & 77 \\
\hline 1998 & 957900 & 9784 & 682 & 771 & 83 \\
\hline 1999 & 970200 & 9967 & 760 & 779 & 95 \\
\hline 2000 & 980800 & 10849 & 747 & 787 & 106 \\
\hline 2001 & 991500 & 12087 & 782 & 857 & 98 \\
\hline 2002 & 1003000 & 12272 & 782 & 854 & 115 \\
\hline 2003 & 1018900 & n.a. & 801 & 810 & 124 \\
\hline 2004 & n.a & n.a & 812 & 831 & 106 \\
\hline 2005 & n.a & n.a & 818 & 775 & 118 \\
\hline
\end{tabular}

\section{Canadian firms that publish}

In the 1980-2005 period, 5,600 firms with a Canadian address published at least one peer-reviewed paper indexed in the SCI database. Interestingly, we found that the number of firms that published scientific papers increased at a $4.2 \%$ compound annual growth rate (CAGR), whereas the number of papers published by the same set of firms increased much more slowly at a CAGR of only $2.7 \%$ (Figure 2$)^{6}$. There are two corollary consequences to these trends: there is a diversification of firms that publish papers; there is a diminishing average number of publications per firm. A detailed analysis of the data reveals that three institutions were largely responsible for the observed decrease in the average number of papers per firm. The publications output of Atomic Energy of Canada Limited (AECL) and HydroOntario, both of which traditionally published on nuclear technology, radically decreased over the years. Also, the publications output of the Canadian subsidiary of Xerox also fell significantly. When these three organisations are removed from the dataset, the number of papers per institution becomes more stable.

Figure 2 Number of papers and number of firms that publish, 1980-2005

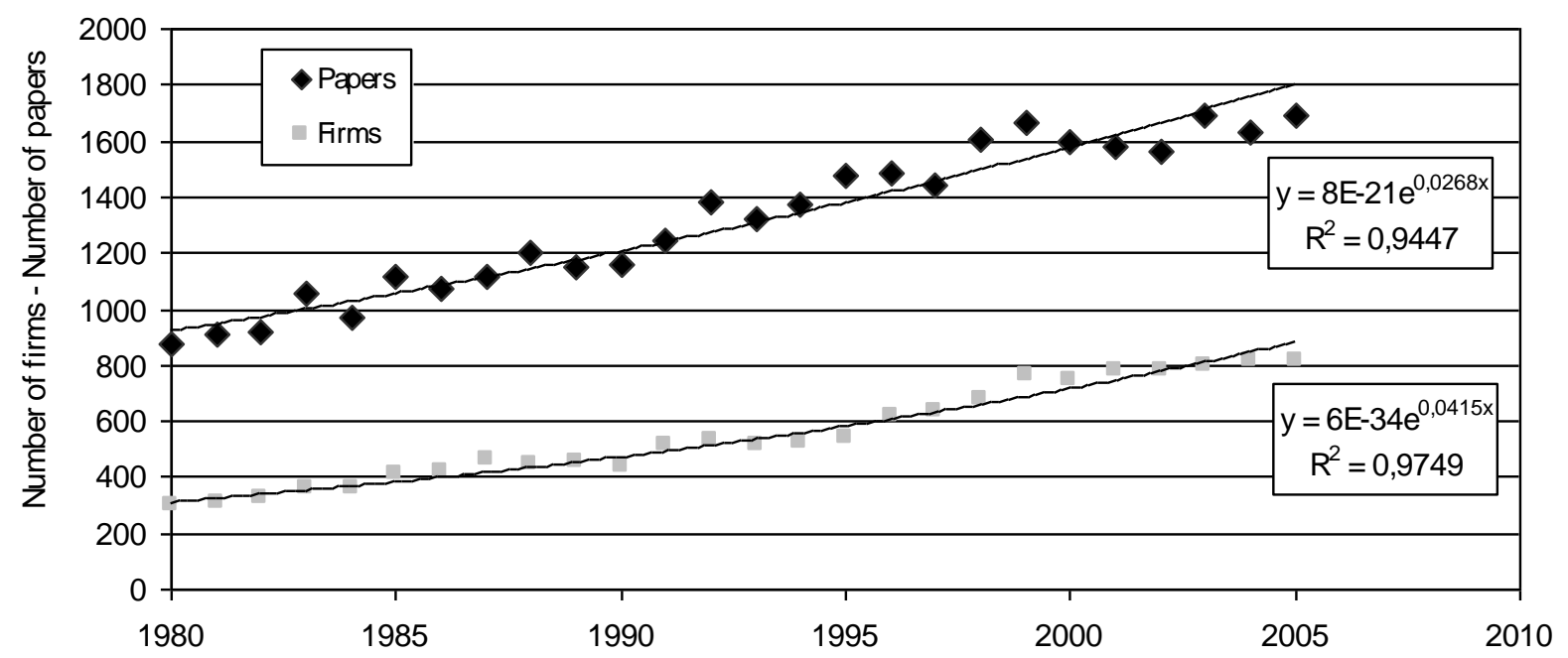

\footnotetext{
${ }^{5}$ Data on the number of Canadian firms active in R\&D are from Schellings (2005); data on the global number of firms in Canada are from Kanagarajah (2006).

${ }^{6}$ These figures were obtained using geometric means; estimating growth using an exponential regression provides essentially the same results.
} 


\section{Canadian firms that hold US patents}

Figure 3 shows that the number of Canadian firms that were granted at least one US patent had a $4.3 \%$ CAGR, and that the number of patents grew even faster with a CAGR of $5.8 \%{ }^{7}$. The shape of the latter distribution is not exponential over the whole time period; it loosely follows an S-shaped curve. The number of patents granted to Canadian firms grew steadily between 1980 and 1997 and, similar to the trend for number of patents granted by the USPTO overall, peaked between 1998 and 2001 after which it reached a steady state but there is a notable decline in the number of patents obtained in 2005. Figure 3 shows that the number of firms obtaining patents increased steadily across the whole period. Overall, the number of patents per firm fell slightly between 1980 and 1997 but subsequently increased at a quite rapid rate.

Figure 3 Number of patents and number of firms that hold patents, 1980-2005

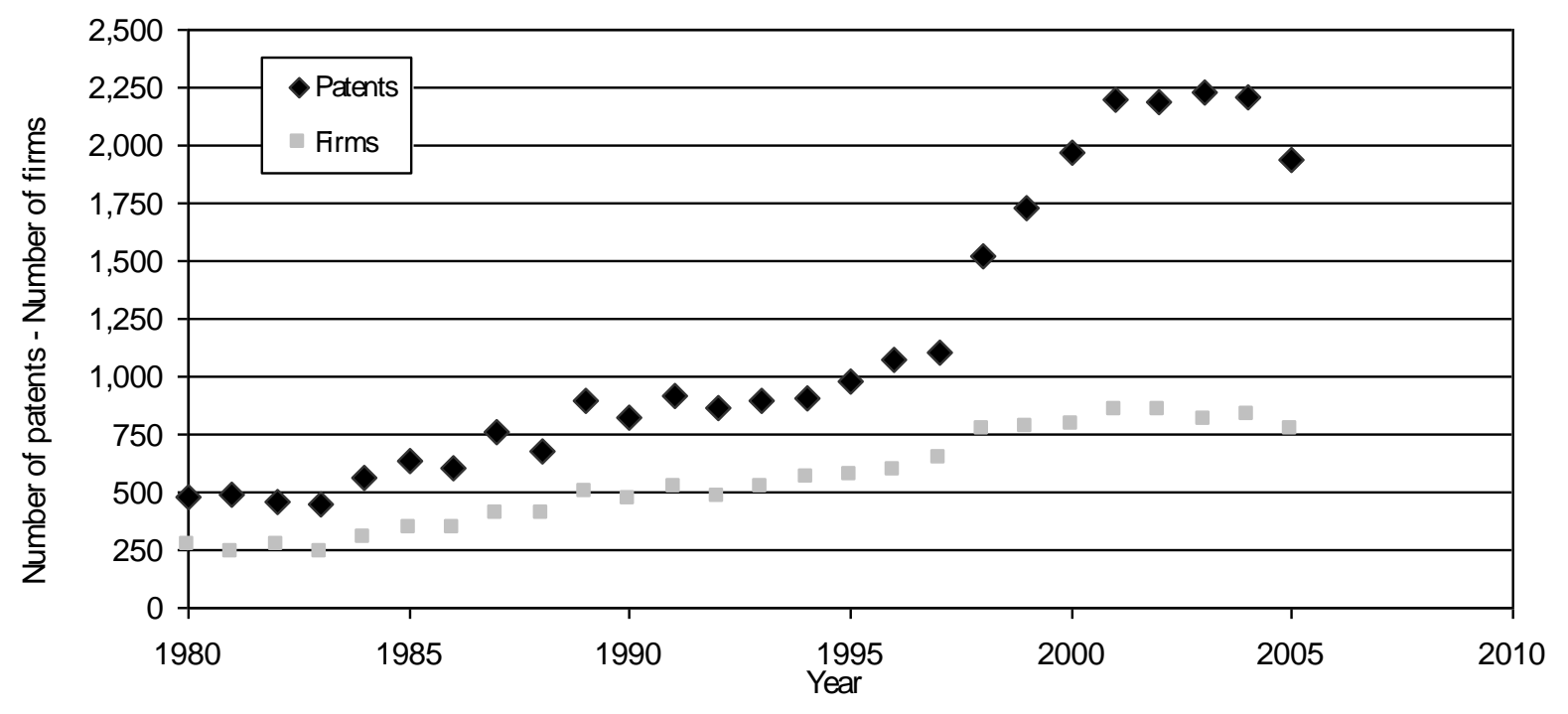

\section{Firms that hold patents and publish papers}

Combining the data on scientific publishing and on patenting produces original results as this type of evidence has rarely if ever been obtained over the whole activity of firms in a country over such a long time period. When examining firms that both publish papers and obtain patents, there is a greater number of papers that were written (approximately 20,700) compared to the number of granted patents (approximately 15,500). These data confirm the findings of other writers (see, e.g., Hicks, 1995; Godin, 1996), that publishing by firms is an important activity, and our data show that in the population of Canadian firms, publishing and patenting are almost equally frequent.

Figure 4 shows that the number of firms that publish and also receive patents any given year grew threefold between 1980 and 2005. It shows that the number of papers produced by these firms was traditionally greater than the number of patents obtained. However, due to a phase started in 1999 recognisable by a marked increase in the number of patents received by Canadian firms and a fairly smooth decline of papers published, the number of granted patents eventually overtook the number of published papers. Over the whole period, for firms publishing papers and receiving patents, the number of papers grew slightly more than twofold, while the number of patents granted to this group of firms increased about fivefold.

The suggestion that firms that are active in scientific publications and patenting are large is supported by the fact that $43 \%$ of the published output is produced by firms that both publish papers and obtain IP protection, although these represent only $17 \%$ of the firms that published scientific papers during the period. Likewise, $51 \%$ of patents are obtained by firms that publish and patent, and these represent a mere

\footnotetext{
${ }^{7}$ These figures were obtained using the geometric mean, estimating growth using an exponential regression provides growth values of respectively $5.5 \%$ and $7.4 \%$ per annum.
} 
$13 \%$ of Canadian firms that hold a US patent granted during the period. When four outliers (Merck Frosst, Nortel Networks, Xerox and Alcan) are removed, the data show that there is a very weak correlation at the firm level between the number of papers published over the period and the number of patents held $\left(r^{2}=0.09\right)$.

Figure 4 Numbers of papers and patents and number of firms, 1980-2005

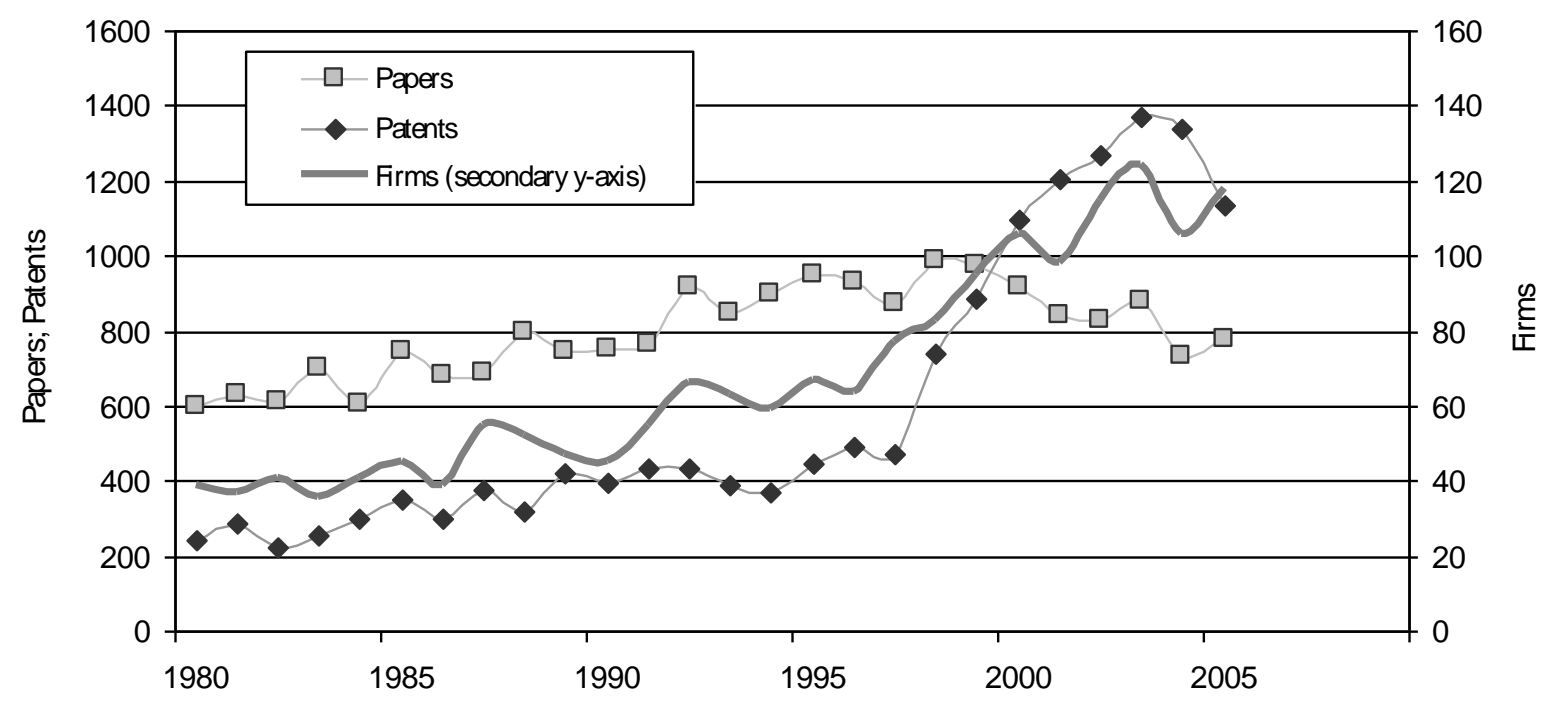

\section{Are firms at the scientific forefront also at the technological frontier?}

Using an indicator that shows whether journals publish basic or applied research, Figure 5 shows that firms that publish papers and obtain patents have a tendency to perform more basic research $(p<0,001)$ than those that only publish. This is not an entirely intuitive result. It might have been expected that firms that only publish would perform more basic research than those that publish and also patent. The observation that firms that publish scientific research and obtain patents appear to perform more basic research than firms that only publish may be reflection of firm size. As we have seen, firms that both publish and patents are more active in publications and in patenting than firms that only publish or only patent which is likely a reflection of their larger size.

Figure 6 shows that firms that both publish papers and hold patents were traditionally cited much more frequently than firms that only published papers. Similarly, Figure 7 shows that firms that both publish papers and hold patents publish in journals that are more frequently cited than firms that only publish papers ( $t$-test on the aggregate yearly figures shows that $p<0,001)$. This is interesting since it might have been expected that firms that concentrate on publishing articles would produce higher quality output than firms that were also involved in the technological side. Thus, these data show that, at the macro level, firms that publish and patent are closer to the scientific forefront than those that only publish. Importantly, the evidence shows this is changing and firms that only published during these years are increasingly producing an output of high quality and, in the latest year studied, this output is cited more than world average-which is shown by increasing levels of citations and a trend towards publishing in more highly cited journals. 
Figure 5 Research level of Canadian firms, 1980-2005

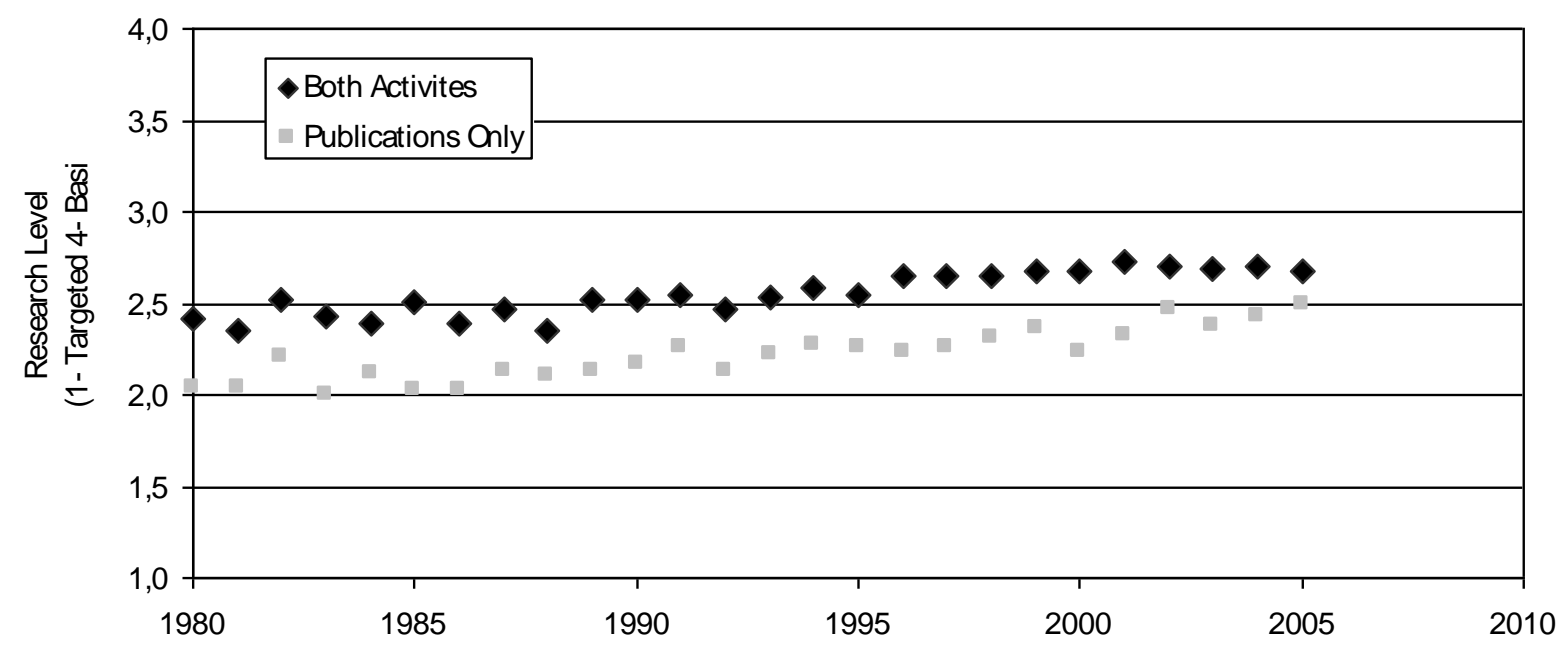

Figure 6 Average of relative citations (ARC) of Canadian firms, 1980-2005

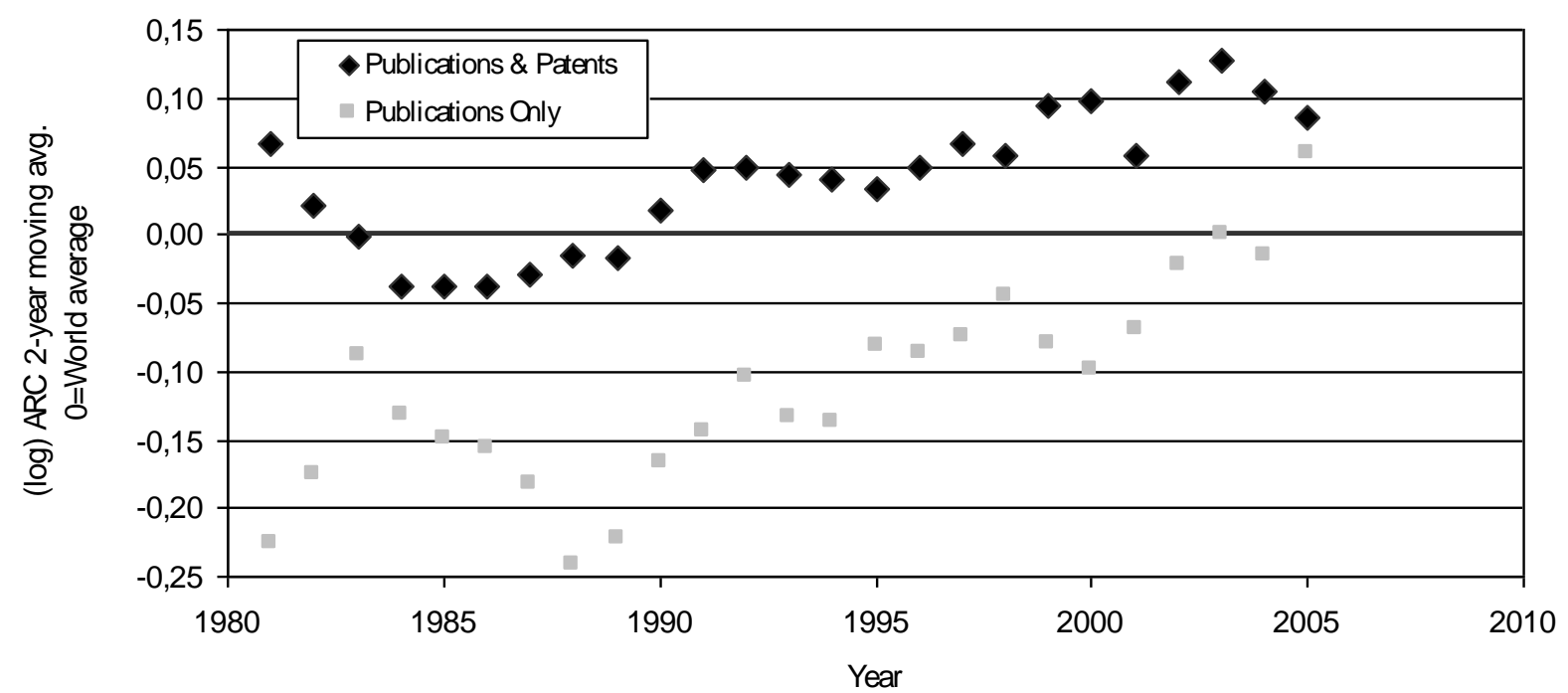

Figure 7 Average of relative impact factors (ARIF) of Canadian Firms, 1980-2005

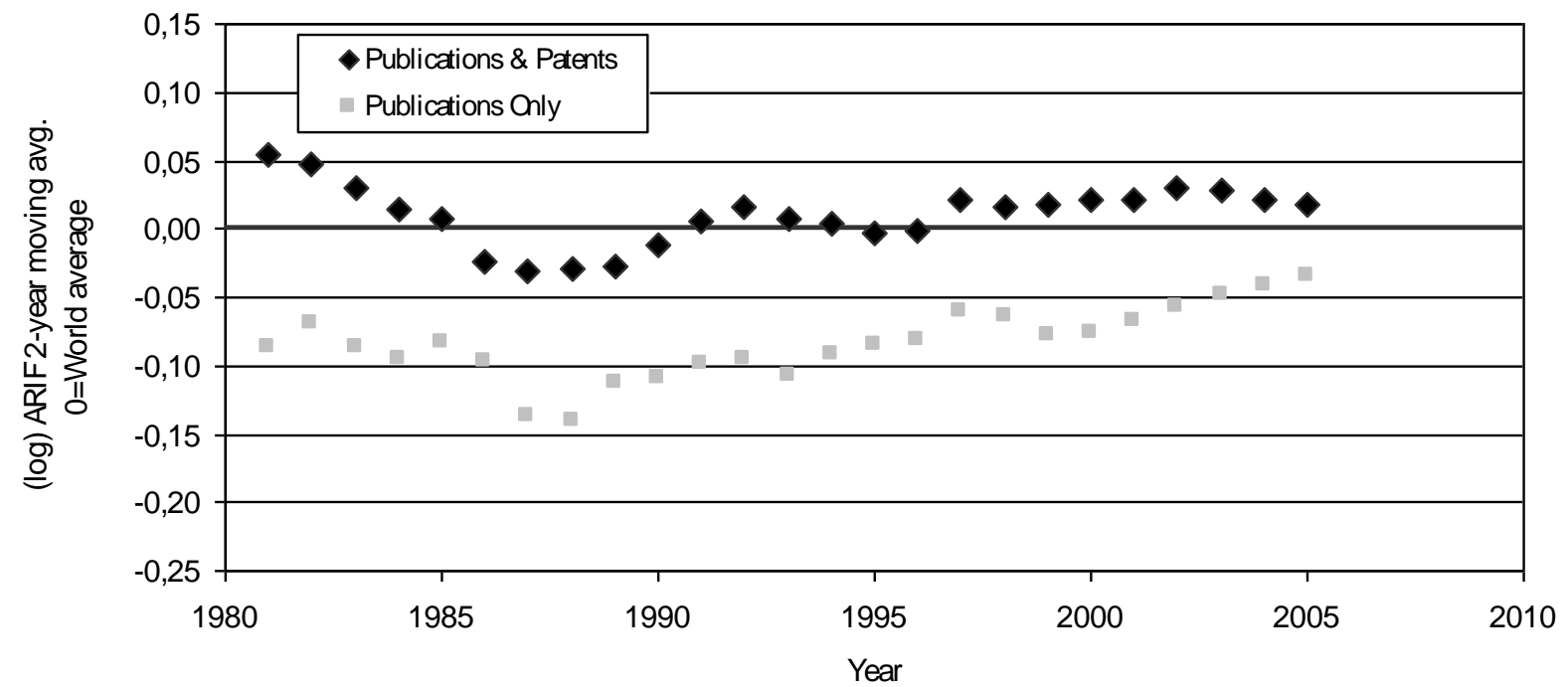

Figure 8 shows that patents held by firms that both publish and patent have, over the entire period, a significantly higher technological impact (1.11 vs. $1.05, p<0,001)$ than patents owned by firms that hold patents but that do not publish scientific papers. This shows that technological research undertaken in 
firms active in both spheres of activities is more likely to be at the technological frontier than that of firms only active in technological research.

Figure 8 Patents average of relative citations (PARC) held by Canadian Firms, 1980-2005

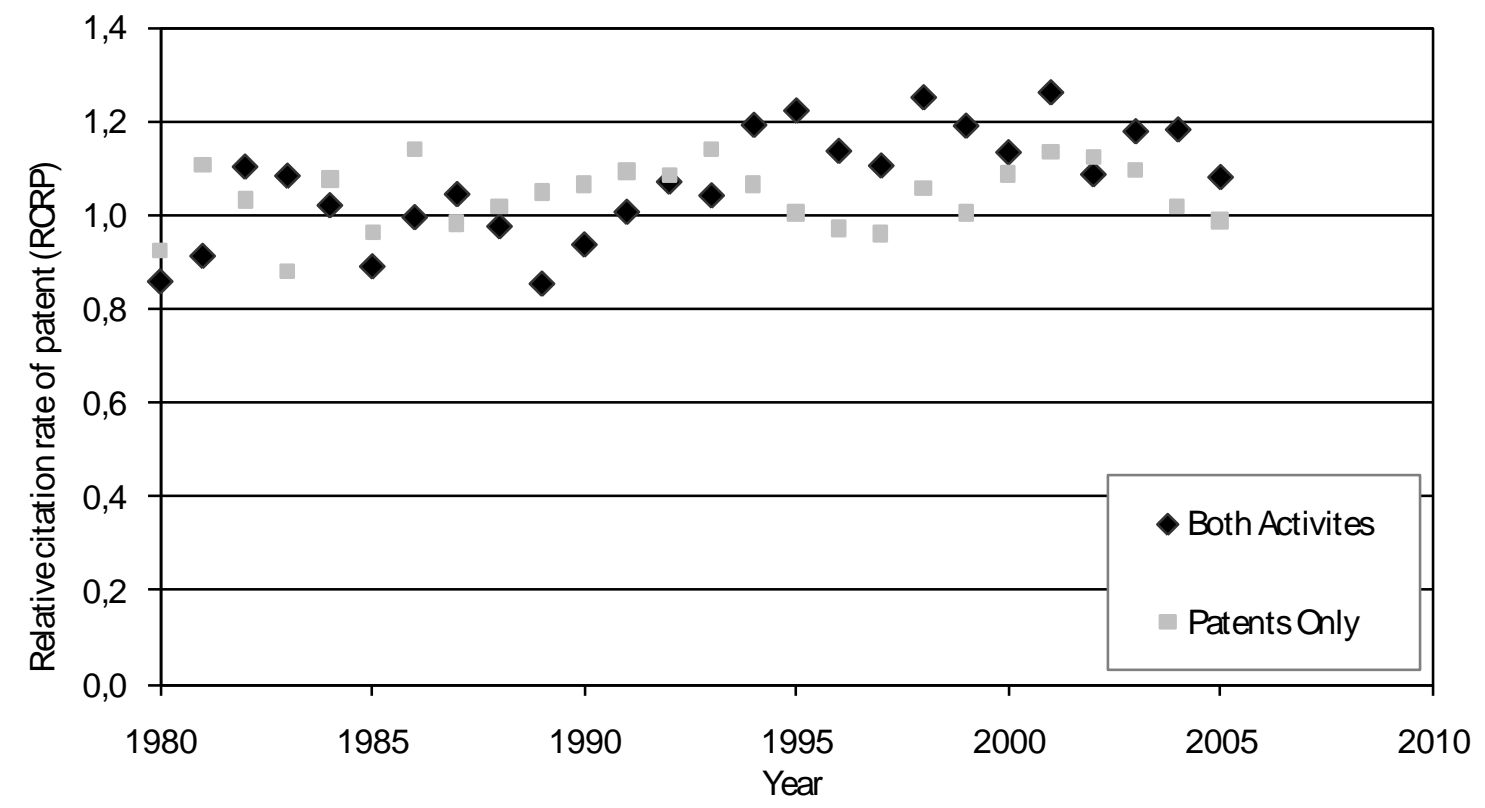

\section{Discussion and Conclusion}

The present paper described scientific output measured by papers and technological output as measured by patents for Canadian firms between 1980 and 2005. The whole population of firms that published at least one paper indexed in SCI during this period and all firms that held at least one patent granted by the USPTO during the period were included. This study clearly shows that the number of firms performing scientific research is increasing regularly. In Canada, the number of firms with a measured scientific output is growing at $4.2 \%$ per annum. The growth in number of firms obtaining a US patent was increasing at essentially the same rate (4.3\% per annum) between 1980 and 2005. Therefore, our results confirm the increasing role played by science and by technology in corporate development.

This paper suggests that it might be more common for larger firms to conduct basic research and obtain patents - this would be expected as both require substantial investments and in many cases only longer terms returns on investments. A cross-examination of the 902 Canadian firms that both patent and publish with the 500 Canadian firms having the largest market capitalization indeed reveals that 50 firms are in both lists. This shows that firms obtaining patents and publishing papers are 60 times more likely than Canadian firms generally to be counted among the large cap companies. Moreover, these 50 companies had a market capitalization which was 3.4 times greater than the average of the 500 largest public firms in Canada and their revenues were 2.9 times larger. A very relevant question is whether this large size is due to their capacity to conduct scientific research and protect their IP or these firms can afford to conduct R\&D because of their large means. Our data does not allow us to address this question but a longitudinal study that would examine the evolution of scientific output, IP protection, and market capitalization over a long time period would shed light on this important question.

This paper suggests that although there is no correlation between the numbers of papers published by firms and the numbers of patents they obtained, overall, firms that perform scientific research and that actively protect their inventions 1) perform research that is more basic than firms that only publish scientific papers, but do not protect their IP with patents; 2) publish in more highly cited journals than firms that only perform scientific research; 3) publish papers that are more highly cited; 4) hold patents that are more frequently cited.

This suggests that, when publishing papers, firms may trade short-term economic benefits in favour of establishing a reputation as leaders which would subsequently be translated into competitive technological advantages. A good reputation can increase innovative capability by enabling firms to hire the best 
researchers and find highly capable partners with whom to collaborate on R\&D projects. Having a good reputation may also facilitate financing and may help to win contracts and sell products and services. The fact that firms that were active both scientifically and technologically published in highly cited journals give credence to the hypothesis that firms publish their scientific results to improve their reputation. Examining the list of firms that published and patented, one has the impression that a substantial proportion of these firms have been subject to takeovers. In the future, examining how small firms use both patents and publications to increase their value could be conducted while examining takeover patterns. In particular, it would be interesting to examine whether firms that are active in R\&D are more frequently acquired than similar firms. It would also be interesting to see if these firms have a different mortality rate.

Our research also reveals that there are two profiles of bibliographic output among Canadian firms, each one comprising about the same number of firms. Some firms have a more scientific profile, while others have a more technological fingerprint. It is noteworthy that there is little overlap between these two types of output and perhaps even more importantly, the vast majority of firms $(99.85 \%)$, and even those reportedly performing $\mathrm{R} \& \mathrm{D}(88 \%)$, neither publish papers nor obtain patents. For the community of practitioners interested in the measurement of S\&T, these results raise important questions since the commonly used output indicators can only measure the tip of the iceberg.

There are several aspects that need to be considered in further research on output indicators for enterprises: 1) it is possible that the majority of firms that are reportedly performing $R \& D$ may not engaged in scientific or technological research, but may rather be engaged in development work, which is not identified by indicators such as papers and patents; 2 ) it would be interesting to verify whether the declaration by Canadian firms that R\&D is being performed may be a potent mechanism to receive tax exemption by firms which actually conduct little real research;3) perhaps that firms reportedly performing $\mathrm{R} \& \mathrm{D}$ tend to be largely unsuccessful in this activity which means they produce little original knowledge that can either be published or patented; 4) it may also be that the majority of firms reportedly performing $\mathrm{R} \& \mathrm{D}$ do not elect to publish or apply for patents as a result of their efforts. Thus, the type of data used in this paper may provide very useful evidence in the definition of $\mathrm{R} \& \mathrm{D}$ and tax policies in addition to explaining how R\&D may increase firms' competitiveness.

\section{Bibliography}

Albert, M B, D Avery, F Narin, and P McAllister 1991. Direct Validation of Citation Counts as Indicators of Industrially Important Patents. Research Policy, 20(1), 251-259.

Amesse, F, C Desranleau, H Etemad, Y Fortier and L Séguin-Dulude 1991. The individual inventor and the role of entrepreneurship: A survey of the Canadian evidence. Research Policy, 20(1), 13-27.

Archambault, É 2002. Methods for using Patents in Cross-country Comparisons. Scientometrics, 54(1), $15-30$.

Archambault, É and V Larivière 2007. Origins of Measures of Journal Impact: Historical Contingencies and their Effect on Current Use. In Proceedings of the 11th Conference of the International Society for Scientometrics and Informetrics (ISSI), eds Torres-Salinas D and H F Moed, pp. 45-51. Madrid: CSIC.

Bar, T 2006. Defensive Publications in an R\&D Race. Journal of Economics and Management Strategy, 15(1), 229-254.

Beer, J J 1958. Coal Tar Dye Manufacture and the Origins of the Modern Industrial Research Laboratory. Isis, 48, 123-131

Beer, J J 1965. The Historical Relations of Science and Technology. Technology and Culture, 6(4), 547552.

Condit, C W 1965. Comment: Stages in the Relationships between Science and Technology. Technology and Culture, 6(4), 587-590.

Furukawa, R and A Goto 2006. The Role of Corporate Scientists in Innovation. Research Policy, 35(1), 24-36. 
Garfield, E 1979. Is Citation Analysis a Legitimate Evaluation Tool? Scientometrics, 1(4), 359-375.

Godin, B 1996. Research and the practice of publication in industries. Research Policy, 25(4), 587-606.

Hall, B H, A B Jaffe and M Trajtenberg 2000. Market Value and Patent Citations: A First Look Economics Department, University of California, Berkeley, Working Paper E00-277. Available at http://repositories.cdlib.org/iber/econ/E00-277

Harhoff, D, J Henkel and E von Hippel 2003. Profiting from Voluntary Information Spillovers: How Users Benefit By Freely Revealing Their Innovations. Research Policy, 32(10), 1753-1769.

Hicks, D 1995. Published Papers, Tacit Competencies and Corporate Management of the Public/Private Character of Knowledge. Industrial and Corporate Change, 4(2), 401-424.

von Hippel, E 1987. Cooperation Between Rivals: Informal Know How Trading. Research Policy, 16(6), 291-302.

Jaffe, A B and M Trajtenberg 2002. Patents, Citations, and Innovations: A Window on the Knowledge Economy. Cambridge, USA: MIT Press.

Kanagarajah, S 2006. Business Dynamics in Canada, 2003. Statistics Canada. Catalogue no. 61-534XWE2006001.

Meyer, M 2000. Does Science Push Technology? Patent Citing Scientific Literature. Research Policy, 29(3), 409-434.

Mowery, D C 1983. Economic Theory and Government Technology Policy. Policy Sciences, 16(1), 29-43.

Muller, P and J Pénin 2006. Why do Firms Disclose Knowledge and How Does it Matter? Journal of Evolutionary Economics, 16(1-2), 85-108.

Multhauf, R P 1965. Sal Ammoniac: A Case History in Industrialization. Technology and Culture, 6(4), $569-586$.

Narin, F, G Pinski and H H Gee 1976. Structure of the Biomedical Literature. Journal of the American Society for Information Science, 27(1), 25-45.

Narin, F, K S Hamilton and D Olivestro 1997. The Increasing Linkage between U.S. Technology and Public Science. Research Policy, 26(3), 317-330.

Nelson, R R 1959. The Simple Economics of Basic Scientific Research. Journal of Political Economy, 67(3), 297-306.

Pinski, G and F Narin 1976. Citation Influence for Journal Aggregates of Scientific Publications: Theory, with application to the literature of physics. Information Processing and Management, 12(5), 297-312

Rosenberg, N 1990. Why Do Firms Do Basic Research (with their own money)? Research Policy, 19(2), $165-174$.

Schellings, R 2005. Industrial R\&D Statistics by Region 1994 to 2003, Statistics Canada. Catalogue no. 88F0006XIE - No. 017.

de Solla Price, D 1965. Is Technology Historically Independent of Science? A Study in Statistical Historiography. Technology and Culture, 6(4), 553-568.

Vinkler, P 2004. Characterization of the Impact of Sets of Scientific Papers: The Garfield (Impact) Factor. Journal of the American Society for Information Science and Technology, 55(5), 431-435. 